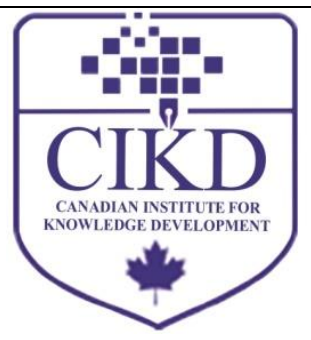

\title{
Transformational Leadership and Neurofeedback: The Medical Perspective of Neuroleadership
}

\author{
Rizki Edmi Edison ${ }^{1}$, Solikin M. Juhro ${ }^{2}$, A. Farid Aulia ${ }^{2}$, Puti Archianti Widiasih ${ }^{3}$ \\ ${ }^{1}$ Neuroscience Center, University of Muhammadiyah Prof Dr HAMKA \\ ${ }^{2}$ Bank Indonesia Institute \\ ${ }^{3}$ Faculty of Psychology, University of Muhammadiyah Prof Dr HAMKA
}

\section{Keywords: \\ Applied Neuroscience, \\ Leadership Style, \\ Transformational \\ Leadership, Human \\ Resources Management, Neurofeedback}

\section{Received}

30 June 2018

Received in revised form

24 November 2018

Accepted

29 November 2018

Correspondence:

rizkiedmiedison@uhamka.ac.id

\section{Abstract}

\begin{abstract}
The world is now facing such a volatile, uncertain, complex, and ambiguous situation known as VUCA that it has generated such new challenges. Therefore, it is imperative that a leader to have competences that always grow. Transformational leadership is a type of leadership deemed superior to anticipate those challenges, where a leader is supposed to always be agile in achieving the organizational goals. This study aimed at analyzing a correlation among leadership aspects (through Multifactor Leadership Questionnaire/MLQ assessment), cognitive functions (through Wechsler Bellevue assessment), and brain activity pattern/brain mapping (through Electroencephalography/EEG). Then, we gave a stimulus through neurofeedback to train the participants' control over their brain waves. The results revealed that all participants that became the research samples had a transformational leadership type with idealized influence (behavior) and inspirational motivation subtypes. Moreover, the presence of stimulation to the brain (neurofeedback) enabled the participants to control their brain waves when they were conscious. The leaders will be able to perform optimally when they can control their brain waves and when they are conscious.
\end{abstract}

(C)AIMI Journals

Changes certainly take place. Heraclitus, a Greek philosopher, stated, "The only thing that does not change is the change itself." Moreover, that quote has become a concept ever since (Muessig, 1969). Changes do not always take place as expected; in fact, in many cases they are unpredictable. The world is now facing a volatile, uncertain, complex, and ambiguous situation known as VUCA, and it triggers a "new normal" condition - a condition when the world is full 
of chaos and turbulence, and it is marked with all aspects of life changing very rapidly (Lawrence, 2013). A condition that requires all institutions, especially institutions in the economic sector, to pay a serious attention to it (Salah \& Watson, 2017).

New emerging challenges require new leadership competences (Lawrence, 2013). Those conditions full of challenges require a tough leader (Radha \& Kosuri, 2017). A leader who possesses strategic foresights to anticipate any changes that may emerge; a leader who is aware of his or her strengths and weaknesses; a leader who is an adaptive, long life learner who is open to changes; a leader who communicates effectively and who is capable of collaborate with others well.

An institution with effective leadership has an opportunity to be a top twenty-percent institution with the best finance three times as much as an institution lacking effective leadership (Axon, Friedman, \& Jordan, 2015). Therefore, we cannot deny the fact that leadership is a very crucial matter needed by all institutions (Charan, 2009).

The term leadership is often confusing and raises many debates (Vince, 2004). One of the main causes is that the leaders' generation gaps result in different characteristics (Salahuddin, 2010). Basically, leadership is defined as the art to move other people to achieve a goal (Naylor, 2003) voluntarily. Leadership is a two-way relationship (Yammarino, 2013); thus a leader has to understand how the brain works as the drive of human activities.

In general, there are three types of leadership namely transactional leadership, transformational leadership, and laissez faire leadership (Avolio \& Bass, 1991). Studies have shown us that the best type of leadership is transformational leadership (Benjamin \& Flynn, 2006) since it is able to enhance the followers' satisfaction the best (Berson \& Linton, 2005). High levels of the followers' satisfaction to their jobs will indirectly trigger the organization to achieve its goals (Bass \& Avolio, 1994).

However, it is not easy to be a transformational leader. Therefore, applied neuroscience is employed to help the transformational leader anticipate those accelerated changes, both the humans themselves and their surroundings. This thing happens since the brain is the master control that integrates the whole body systems including the main components of feelings and instincts. A leader is the master control in his or her organization. He or she can control other people's brains by controlling his or her brain in order that his or her followers' brains can resonate with their leader's brain (Boyatzis, Smith, \& Blaize, 2006).

Of all branches of neuroscience, social cognitive neuroscience - which understands the human brain's mechanism when interacting with others (Ochsner \& Lieberman, 2001) - has been applied in the organization the most, especially in the field of leadership (Waldman, Balthazard, \& Peterson, 2011). The term neurological leadership is defined as an applied social cognitive neuroscience employed to analyze and understand leaders' behaviors (Liu, Ying, \& Gau, 2015).

Studies on how the brain affects human behaviors were first conducted on an accident case at work to Phineas Gage, a construction worker ${ }^{1}$, in 1848 (Haas, 2001). The findings revealed that a brain part called lobus frontalis on the left was damaged. That part of the brain served to

\footnotetext{
${ }^{1}$ Phineas Gage got an accident while setting up explosives to build a railway infrastructure. The explosives exploded before he managed to hide in a safe place. A piece of iron chunk impaled his left chin, entering the skull, past the eyes towards the brain, penetrating his left, front head. Phineas survived after the surgery to remove the chunk. However, his attitudes changed drastically. He totally had different attitudes and behaviours after the accident (Haas, 2001).
} 
think rationally (Siddiqui, Chatterjee, Kumar, Siddique, \& Goyal, 2008). These findings have proven that the human brain can affect one's attitude and behaviors (Kolb \& Whishaw, 2011).

Neuroscience recognizes what is called as neuroplasticity and neurogenesis. Neuroplasticity is a condition where the brain adjusts itself with habits that are usually done (Rosenzweig \& Bennet, 1999) or a condition where the healthy brain takes over parts of diseased brain (Murphy \& Corbett, 2009). Neurogenesis is a condition where new nerves on the brain are generated (Sierra, Encinas, \& Maletic-Savatic, 2011). Studies have shown that the human brain can grow and develope at any age (Ming \& Song, 2012). Doing aerobics (GomezPinilla \& Hillman, 2013) and fasting (Cherif, Roelands, Meeusen, \& Chamari, 2016) regularly will enhance the quality of hippocampus, part of the brain that serves as a memory storage. The example mentioned above shows that the brain can affect behaviors, and the other way around, behaviors can affect the human brain.

A study conducted by Juhro and Aulia (2017) concluded that applied neuroscience was a relatively outstanding approach in explaining effective transformational leadership behaviors. Nevertheless, that study has not been supported with empirical exercises, especially physical tests on the relations between the real-time brain activities and the transformational leadership behaviors. Therefore, the present study was the continuation of Juhro and Aulia's (2017) study in relations to the roles of applied neuroscience in supporting the performance of transformational leadership. This study was aimed at finding out a transformational leader's brain mapping and the impact of stimulations employed in the neuroscience approach, such as neurofeedback, in altering the shape of a leader's brain activities. Of the objectives of the study that we would like to achieve, we really hope that this study can benefit us with the presence of objective indicators that can state whether one is a transformational leader or not. We hope that our best efforts to encourage this applied neuroscience-based transformational leadership shall positively impact on it as a reliable alternative method.

Based on the results, it can be concluded that all participants are transformational leaders with idealized influenced (behavior) and inspirational motivational leadership subtype. Furthermore, this study showed that the stimulation of the brain (through neurofeedback) make participants able to control their brain waves in a conscious state. A leader is expected to work more optimal if they can control their brain waves.

\section{Literature Review}

The world is now showing situations called VUCA (volatility, uncertainty, complexity, and ambiguity) (Lawrence, Steck, \& Pryplesh, 1991). The term was used for the first time by military institutions (Stiehm, 2002). The term VUCA started to be used in the economic sector after a global financial crisis broke out in 2008-2009 (Tovar, 2016). We have felt these VUCA situations for the past decades; as a result, how we perceive economic conditions has also changed (Sarkar, 2016). One instance of VUCA conditions was The United Kingdom's referendum decision in 2016 to exit from the European Union, known as British Exit (Brexit). Those conditions resulted in that country's long-term economic planning disorder (Hunt \& Wheeler, 2017). VUCA that can emerge from many sides has stimulated leaders to be wise and agile when facing those conditions. 
Leadership is a multi-level interaction involving several factors such as a leader, followers, and inter-related situations (Hollander, 1984). Burns (1978) categorizes leadership into two types namely transformational leadership and transactional leadership. Transactional leadership is based on a give-and-take principle and is limited to rewards and punishments between the leader and the followers (Podsakoff, Bommer, Podsakoff, \& Mackenzie, 2006). Meanwhile, transformational leadership prioritizes more on the followers' participation as part of the group to achieve a common goal (Weiherl \& Masal, 2016).

Furthermore, Bass and Avolio (1991) developed Full Range Leadership theory elaborating leadership concepts namely transformational leadership, transactional leadership, and laissezfaire leadership. Transformational leadership consists of idealized influence (attributed), idealized influence (behavior), inspirational motivation, intellectual stimulation, and individualized consideration. Transactional leadership consists of contingent reward, management-by-exception (active) and management-by-exception (passive).

An effective leader can encourage his or her organization to achieve its targets (Sarin \& McDermott, 2003), while an ineffective leader will hamper his or her organization in achieving its targets (Bradley \& Herbert, 1997). Transformational leadership is the most effective leadership type of all (Xu \& Wang, 2008). This type also enhances the followers' satisfaction when they work (Podsakoff et al., 2006). As a result, their work performance is enhanced, and it positively impacts on the organizational performance (Harter, Smith, \& Hayes, 2002).

Neuroscience is a branch of science that discusses the human brain and its effects on human behaviors (Garret, 2003). Neuroscience discusses neuron interactions in the brain, the relations between the neurons and the blood vessels, neuron reactions on the stimulus, the impact of neurons on an individual's decision-making process (Augustine, 2004). In the development of neuroscience, there are some challenges to integrate those existing branches of science in order that our understanding on the human brain can be applied in various practical aspects of life, such as marketing, education, politics, and leadership. Social cognitive neuroscience is a branch of neuroscience deemed the most possible to be applied in the fields of leadership (Ochsner \& Lieberman, 2001). These inter-disciplines of science study an individual's interactions with other individuals or in a certain situation. Neurological leadership or neuroleadership can be deemed as an application of social cognitive neuroscience to understand and analyze leaders' attitude and behaviors (Liu et al., 2015).

\section{Brain Leadership Assessment}

In this study, we assessed a leader's leadership characteristics through two approaches, questionnaire-based and brain mapping based. There are two tests in questionnaire-based approach which assessed leadership type (through MLQ) and cognitive function capability (through WB). Brain mapping based (through EEG) approach was employed to understand a leader's patterns of his or her brain activities (Waldman et al., 2011).

\section{Questionnaire-Based Leadership Assessment}

One questionnaire-based approach conducted was Multiple Leadership Questionnaire (MLQ). MLQ is an instrument used to assess transformational leadership and transactional leadership behaviors (Bass \& Bass, 2008). MLQ developed by Bass and Avolio (1994) comprises 
assessments of five transformational leadership subtypes, three subtypes of transactional leadership and one subtype of laissez-faire leadership.

In transformational leadership, there are five subtypes namely: 1) Inspirational motivation, A leader's articulation capability. A leader who positively envisions the future will make his or her followers motivated, 2) Idealized influence (attributed), it is attributed to a leader's charisma. That positive attribute will make his or her followers be able to nurture their strong emotional relations and trust to their leader, 3) Idealized influence (behavior), it stresses on collective awareness of the mission and the values, 4) Intellectual stimulation, it stimulates the followers to have an assumption on a problem actively and anticipatively, and 5) Individual consideration, it refers to an effort to put the followers' needs and capabilities into consideration.

In transactional leadership, there are three subtypes namely 1) Contingent reward, a leader's behaviors that focus on tasks given with material or moral rewards if the tasks are well carried out, 2) Active management-by-exception, a leader's behaviors that observe events taking place in the organization in accordance with the rules and regulations and avoid things that do not comply with the rules and regulations, and 3) Passive management-by-exception, a leader will intervene if he or she finds any mistakes and will conduct an evaluation if things do not go by the book.

Moreover, in laissez-faire leadership, the subtypes discuss absence of leadership. The leader does not seem to take part in the organizational activities and does not pay attention to any situations that require a quick decision making. He or she will simply leave all operational matters to the followers.

Then, we conducted the second test namely Wechsler Bellevue Test. This test was used to assess one's cognition. This test can assess cognition of an individual older than ten years old (Rabin \& Guertin, 1951). We measured two kinds of scales namely verbal and performance. In details, this test consisted of information test, general comprehension test, memory span test, arithmetical reasoning test, similarities test, vocabulary test, block design test, object assembly test, and digit symbol test.

\section{Brain-Mapping Based Assessment}

Recording a human brain's activities with EEG was conducted for the first time by a scientist from Germany, Hans Berger, in 1924 (Stone \& Hughes, 2013). Recording brain's activities through recording electric pulses in the brain was beneficial in many cases, such as detecting abnormalities in the human brain before CT Scan and MRI were invented (Tudor, Tudir, \& Tudor, 2005). 10-20 international system of EEG electrode placement was conducted in the EEG electrode laying process on the human brain (Homan, Herman, \& Purdy, 1987).

The next stage was neurofeedback. The concept of neurofeedback has been an object of studies for the past decades. Neurofeedback is a tool that can be employed to find out the human brain's activities. Neurofeedback can also help us train our brain's activities (Heinrich, Gevensleben, \& Strehl, 2007) by controlling the brain wave when we are conscious.

In outline, there are several types of brain waves namely delta $(1-3 \mathrm{~Hz})$, theta $(4-7 \mathrm{~Hz})$, alpha (8-12 Hz), and beta (13-30 Hz) (Sucholeiki, 2017). The first component of neurofeedback is a stimulus given to the subject. That stimulus can be given to the subject in 
the form of audio, video, or both of them. The next component is electrophysiology component. During the procedure, the subject will realize the changes that are taking place and will be capable of assessing his or her progress to achieve optimal performances. One of neurofeedback treatments is to ask the subject to enhance his or her brain activities based on the changes found in the sound or film stimuli. Several neurofeedback protocols commonly used are alpha, beta, delta, theta, and gamma waves or a combination of those waves such as alpha/theta ratio, beta/theta ratio, and so on (Dempster, 2012).

\section{a. Alpha Protocol}

Alpha waves are often associated with conscious relaxation (Evans \& Abarbanel, 1999). Alpha waves generate the feelings of tranquil and comfortable. Various studies have shown that in general, alpha waves increase when one is meditating. Moreover, all frequencies in alpha waves represent brain activities related to creativity. Alpha waves emerge and are widespread on the skin surface. The most common frequency of alpha waves is $7-10 \mathrm{~Hz}$, usually employed to meditate, sleep, and reduce stress and anxiety. The $10-\mathrm{Hz}$ frequency also relaxes muscles, reduces pain, regulates respiration, and increases the heartbeat (Dempster, 2012; Vernon, 2005). Moreover, at a $10.2-\mathrm{Hz}$ frequency, alpha waves can help enhance memory, enhance mental performances, and heal a brain trauma.

\section{b. Beta Protocol}

Beta activities are good indicators to observe mental performances. Bad beta activities can represent physical or mental disorders such as depression, ADHD, and insomnia (Egner and Gruzelier, 2004).

\section{c. Delta Protocol}

These waves are the slowest waves associated with phase- 3 and phase- 4 sleeps (Surmeli \& Ertem, 2007). These waves can also reduce anxiety and enhance the quality of sleep. Delta waves are employed to ease headache, brain trauma/injury, learning disorder, and to heal severe brain contraction (with a $1-3 \mathrm{~Hz}$ delta waves simulation) (Marzbani, Marateb, \& Mansourian, 2016).

\section{d. Theta Protocol}

Theta waves are related to various brain activities such memories, emotions, creativity, sleep, meditation, and hypnosis. These waves are associated with the early stage of sleep namely when one lightly sleeps and is easily waken up. Theta waves can reduce anxiety, depression, daydreaming, trance, emotional disturbance, and ADHD (Beatty, et al., 1974; Vernon, 2005).

\section{e. Gamma Protocol}

Gamma waves have the highest frequency and are associated with cognitive processing and memory (Staufenbiel, Brouwer, Keizer, \& Van Wouwe, 2014). The speed to recall an event or a thing will increase if these waves have a faster rhythm. This fast rhythm is responsible for neuron connections in the brain and their data transfer to the outer body. Many scientists have studied these waves in hippocampus (part of the brain that serves to convert long-term memory 
into the short-term memory). Gamma wave training is conducted to improve cognition, mental sharpness, brain activities, and problem-solving activities. Not only do these waves enhance calculation skills, but they also enhance the speed of information process, short-term memory, and reduce migraine (Vernon, 2005).

\section{Neurofeedback}

In general, there are two classical guides in the neurofeedback training. They focus on low frequencies (alpha or theta) to enhance relaxation and focus (Gruzelier, 2009) or high frequencies (low beta, beta and theta) to strengthen activation, to organize and to block disturbances (Ros et al., 2009). Neurofeedback is a type of biofeedback employing the real time brain's activities outlook. It generally employs EEG to regulate the brain functions independently. Other information obtained from biofeedback can be a heartbeat, body temperature, and muscle activation. For the past years, people have employed neurofeedback when handling a disease or brain disorder, such as attention deficit/hyperactivities disorder (ADHD); schizophrenia, sleep disorder; learning difficulties, dyslexia, and dyscalculia; drug abuse; autistic spectrum disorder; epilepsy; depression; anxiety; and pain management (Marzbani et al., 2016).

Many experts have developed brain-mechanism based leadership from time to time. They have started to employ neurofeedback in the field of leadership (Waldman, Balthazard, \& Peterson, 2011). Personal development obtained from neurofeedback have been put into consideration to observe the effectiveness of neurofeedback in the fields of leadership. Moreover, experts have often employed neurofeedback as a therapy in cases related to the medical world such as ADHD, depression, anxiety, addiction, and even Parkinson (Marzbani et al., 2016).

\section{Method}

As depicted in Figure 1, this study was conducted based on the framework of three aspects of leadership namely 1). leadership aspects, 2) cognitive function, 3) brain activities pattern/brain mapping, and 4) The impact of neurofeedback on those three aspects.

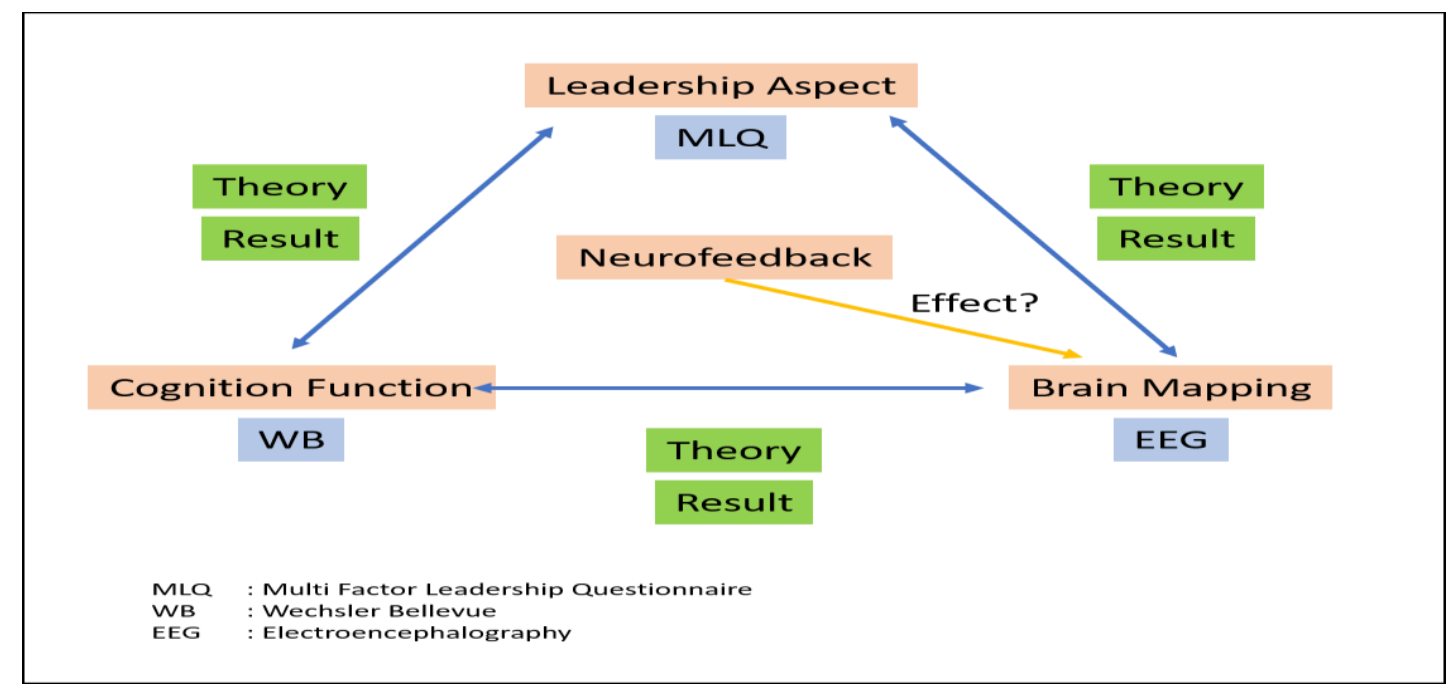

Figure 1. Framework of leadership, cognition function, brain mapping and neurofeedback interlinked 
The framework was aimed at observing the relations among all those leadership aspects (through Multi Factor Leadership Questionnaire), cognition function (through Wechsler Bellevue Test) and brain activity pattern/brain mapping (through Electroencephalography). On the next stage, we gave the stimulus through neurofeedback to observe the brain control over the stimulus given.

In the Multi Factor Leadership Questionnaire test, the participants were interviewed by a psychologist in order to find out their type of leadership style whether it was transactional or transformational. The Wechsler Bellevue tools were employed to examine the participants' cognition especially their intelligence (verbal and performance IQ). Electroencephalography was employed with lobus frontalis as the area of interest with main waves of alpha to record the brain's activities at the neurofeedback stage, the participants were trained to control their own brain waves. The participants were given stimulation in five sessions and then their brain's activities were reexamined with EEG.

There were ten participants in this study. They were managers and over the manager position at Bank Indonesia Institute who had led an organization for more than three years. The exclusive criterion that we stressed upon was that they had no history of a brain disease or a brain trauma. Then, the participants who had understood the benefits and the procedures of the study were asked to sign the inform consent.

MLQ and WB Test Data were obtained through personal interviews with a psychologist. The duration of interviews ranged from15 to 30 minutes (MLQ) and from 60 to 120 minutes (WB). Then, EEG electrodes were installed in accordance with 10-20 international system EEG electrode placement method.

The electrodes installed on the head covered Fp1, Fp2, F3, F4, F7, F8 (region frontal), T3, T4, T5, T6 points (region temporal), C3, C4, P3, P4, O1, O2, Fz, Cz, Pz, A1, and A2 points. Data recording was then conducted in the resting state situation, a situation where the participants were not given any tasks during the data recording. The brain's activities were recorded for 12 minutes. First, they did the base line for one minute. Secondly, they closed their eyes for two minutes. Thirdly, they opened their eyes for two minutes. Fourthly, they closed their eyes for two minutes. Fifthly, they opened their eyes for two minutes. Sixthly, they closed their eyes for two minutes. Last, they did the base line again for one minute. During the data recording, the participants were not supposed to sleep. The data recording was conducted in two stages; first, before the neurofeedback stimulus was given, and second, after the neurofeedback stimulus was given.

In the case of neurofeedback stimulus, the procedures before the electrodes were installed were the same as that of EEG. However, the electrodes employed were only put on C3 and C4, as well as the reference electrodes on the left and right. It took around twenty minutes to give neurofeedback stimulus for each session. Each participant underwent five sessions of stimulations.

\section{Analysis}

We conducted this study to ten participants with the criteria that mentioned above. All of the participants underwent all of the series of the study. 


\section{Multiple Leadership Questionnaire (MLQ) and Wechsler Bellevue (WB) Test}

The results related to MLQ and WB are presented in Table 1.

Table 1

Results of $M L Q$ and $W B$

\begin{tabular}{ccccccc}
\hline Initial & Sex & Age & Leadership Type & Leadership Subtype & V-IQ & P-IQ \\
\hline A & M & $51-55$ & Transformative & Idealized Influence (Behavior) & 60 & 53 \\
B & F & $46-50$ & Transformative & Idealized Influence (Behavior) & 62,5 & 51 \\
C & M & $46-50$ & Transformative & Inspirational Motivation & 62,5 & 46 \\
D & M & $41-45$ & Transformative & Inspirational Motivation & 62 & 55 \\
E & M & $36-40$ & Transformative & Inspirational Motivation & 61 & 65 \\
F & M & $31-35$ & Transformative & Idealized Influence (Behavior) & 65,8 & 61 \\
G & M & $46-50$ & Transformative & Inspirational Motivation & 55 & 58 \\
H & F & $46-50$ & Transformative & Inspirational Motivation & 64 & 57 \\
I & F & $36-40$ & Transformative & Inspirational Motivation & 55 & 60 \\
J & M & $31-35$ & Transformative & Idealized Influence (Behavior) & 64 & 62 \\
\hline
\end{tabular}

The results obtained from MLQ assessment indicated that all of the participants had the characteristics of transformational leadership. Of five subtypes of transformational leadership, four participants had an idealized influence (behavior) subtype, and six participants had an inspirational motivation subtype. Idealized influence refers to collective understandings on the common goals and values, and has behaviors supporting those values. Inspirational motivational stresses on the articulation and vision representation of a transformational leader. A transformational leader of this subtype is capable of perceiving the future with positive attitudes, thus his or her followers will also become motivated (Rowold, 2005).

\section{Electroencephalography (EEG)}

In this study, ten participants underwent their brain activity recording in the resting state condition. The recording took place for twelve minutes. From that recording, the data were filtrated in order to obtain the brain waves without $\operatorname{artifact}^{2}$ when the participants opened their eyes. Those selected brain waves would further be analyzed using the power spectra.

The regions of interest of the brain activities analyzed were frontal regions (Fp1, Fp2, F3, F4, F7, and F8), and temporal regions (T3, T4, T5, T6). Based on the analysis, we did not find any significant differences among the participants in the resting state condition both before and after the neurofeedback. Similar brain activities among the participants supported the previous results obtained from the MLQ and Wechsler Bellevue assessments all of whom were transformational leaders. From both of the two psychological test instruments as well as EEG, we drew an understanding that the brain activities had many things to do with one's type of leadership. This study was in accordance with the study conducted by Waldman, Balthazard, and Peterson (2011) on the relations between the level of the brain coherence and leadership behaviors. The results of the study showed that the brain activities on a certain region determined the leadership characteristics perceived by the followers.

\footnotetext{
${ }^{2}$ Brain wave structure that appeared unnaturally and was caused by a manipulation when brain activities were recorded.
} 
Defoe (2012) also showed that there were relations between the behaviors and the quality of inspirational leadership with a certain pathway in the human brain. Furthermore, it showed that there were two different pathways namely the inspirational leadership pathway that was related to the ability to communicate the visions, altruism, social responsibility, and that focused on serving the people. The pathway contrary to those matters showed the opposite behaviors, namely personalized vision that only accommodated that leader's visions. Therefore, we could draw a conclusion that one's brain activity patterns were related to his or her behaviors or leadership type.

\section{The Relations between Age/Generation and Leadership}

For the past couple of years, many experts have conducted various studies on management and leadership matters focusing on the issues of diversity at the workplace. The most studied diversity issue is generally related to the generation gaps. Scientists have concluded that the impact of generation gaps on the organizational effectiveness is an important aspect to be understood. That understanding is expected to encourage a harmony at the workplace, respectful behaviors, and teamwork. Those matters will eventually impact on the organizational success.

That exposition has based this study to explore the relations between the generation gaps and the leadership type. In this study, those ten participants were categorized into three age groups namely 30-40 years old, 40-50 years old and over 50 years old. The results showed that all of the participants from those three age groups had the same leadership type. In regards to the age factor, there are age differences among the participants. However, a generation is not based on the age difference but also on the great events and environmental changes that take place (Zemke Raines, \& Filipczak, 2000). Jurkiewicz (2000) also states that empirical evidences that show any significance on different leadership type based on the generation or the age group are still too few. A study conducted by Jurkiewicz (2000) has also shown that there are no significant differences between the generation gap and the leadership type. What affects the leadership type more are the recruitment process, retention, motivation, training, and other human resources processes. Tolbize (2008) also similarly reported that not only one's behaviors are determined by the generation factor, but they are also determined by the level of education, the type of jobs, the income, and the marital status.

The results of this study revealed that there were no leadership type differences among the age groups. These results were also supported by the analyses and various theories mentioned above. The same environment and human resources development undergone by those ten participants could affect the participants' same leadership type. Moreover, other things that were not analyzed in this study such as the level of education, the marital status, the type of jobs could have affected the similarity in the participants' leadership type too.

\section{The Relations between Intelligence and Leadership Type}

In this study, the level of intelligence was measured by employing the Wechsler Bellevue (WB) test. The WB test was deemed the best since it had been validated and could be applied to adults. Aspects assessed from the participants included vocabulary, similarities, information, 
comprehension, arithmetic, digit span, letter-number sequencing, picture comprehension, block design, matrix reasoning, digit symbol-coding, and symbol search.

The results of those ten participants' tests showed that there were no significant differences among the participants whether it was the verbal IQ, the performance IQ or the total IQ. All of the participants achieved IQ scores above the average. These results were in accordance with a theory stating that the level of intelligence had many things to do with the leadership type since according to the MLQ assessment, all of the participants had a transformational leadership type.

Several literature reviews discussing the effectiveness of leadership stressed on the importance of intelligence factor (Bass, 1990; House \& Aditya, 1997; Kirkpatrick \& Locke, 1991; Mann, 1959; Stogdill, 1948). Furthermore, Kirkpatrick and Locke (1991) noted that cognitive skills were a leader's important asset since a leader had to be capable of collecting, integrating, and interpreting information in a great amount. A leader has also to be responsible for certain tasks such as formulating a strategy, solving a problem, motivating his or her employees, and monitoring the workplace. Those competences were part of the intelligence functions (Fiedler \& Garcia, 1987). Moreover, creativity is also another mechanism that connects intelligence and leadership (Jung, 2001). Based on the results of WB tests and the analyses conducted in accordance to the theories mentioned above, it can be concluded that there is a relation between the intelligence factor and the leadership type.

\section{The Relations between Leadership and Brain Mapping}

In order to discover empirical evidences between leadership and the brain's activity patterns, we analyzed the relations of those matters. We analyzed the leadership type by employing WB test and we analyzed the brain's activities by employing brain mapping.

A tool that could be employed to emphasize the relations between neurological activities and leadership behaviors was electroencephalography (EEG) technology. Although no experts had conducted a specific research regarding those matters, EEG was deemed capable of predicting the relations between a leadership type and the brain's activities. This was proven by EEG's capability to observe human behaviors in relations to the brain function association.

The results of EEG showed that there were no different brain waves among the participants. All of the participants showed the same brain activity patterns. According to the participants' same brain activity patterns and the participants' same leadership type, we drew a conclusion that a leadership type had to do with the brain activity patterns. The same leadership type was able to predict the same brain activity patterns, and the other way around. This had been proven by various literature studies mentioned above.

Various literature showed that transformational leadership behaviors had to do with the brain's neurological activities (Cacioppo et al., 2003). However, a leader's complex behaviors could not simply be associated with a certain brain's location (Cacioppo et al., 2003; Cacioppo, Berntson, \& Nusbaum, 2008; Lieberman, 2007; Nolte, 2002). The main principle on the brain complexity deemed relevant to a study on transformational leadership was the brain's lobus frontalis and lobus temporal that played an important role in predicting an effective leader's behaviors. Lobus frontalis was responsible for the executive functions such as self-regulation, such as programming, settings, and process verification to understand sensory information in 
planning and regulating behaviors (Lewis, 1997). This directly had to do with transformational leadership behaviors (Case, 1992; Fuster, 1999).

Moreover, it was lobus temporal that played an important role in terms of memory, perception, language and personality (Damasio, 1989). This competence significantly impacted on a leader's effectiveness in anticipating leadership challenges (Hannah, Woolfolk, \& Lord, 2009). Lobus temporal also served to balance psychobiological condition and interactions with other people and his or her environment (Craig, 2009; Schore, 1994). Since transformational leadership required high social perception skills (for example in individualized consideration) and the ability to think logically (for example in intellectual stimulation), lobus temporal played an important role in those functions.

Based on the participants' same leadership type and the participants' brain activity patterns, we can draw a conclusion that there is a relation between the leadership type and the brain activity patterns observed through the brain mapping. Specifically, the leadership type that can be analyzed in this study is transformational leadership.

\section{The Relations between Intelligence and Brain Mapping}

There are three main connectivity types in the brain. First, it is a structural connectivity measured with a structural MRI and Diffusion Tensor Imaging. The connectivity in this level looks exactly the same to a person whether he or she is still alive or right after he or she dies, and it represents the essence of the brain's structures and infrastructures. Second, it is a functional connectivity measured with electroencephalography (EEG) coherence and fMRI correlations between one part of the brain with the other. In this level, temporal correlations between one or more than one part of the brain are measured, and they indicate functional activities owned by those related parts. Third, it is an effective connectivity that measures the size and information pathway curse between two connected parts of the brain or among more than two connected parts of the brain analogically, a structural connection is like a street connecting a parking lot and a sports stadium. Then, a functional connectivity is the correlation between the differences on those two locations, and an effective connection measures the course and the flow size of the people heading towards those locations.

The results of recording in this study indicated that brain activity patterns dominated by alpha and beta waves on all of the participants' lobus frontalis. In this study, the level of intelligence measured by the Wechsler Bellevue tests showed the same extent of intelligence among the participants, namely above average. According to the participants' same brain activity patterns and the participants' same level of intelligence, we could observe a relation between the brain activity pattern and the level of intelligence. In this study, it was also found that a generation gap or different age groups did not affect the brain waves if the participants had the same level of intelligence and the same leadership type.

\section{The Impact of Neurofeedback}

Neurofeedback is a tool that can be employed to train a person to control his or her own brain functions. The effectiveness of neurofeedback can be observed by measuring the brain waves. Although we theoretically know that neurofeedback has many positive impacts, there are still very few empirical evidences that confirm those things (Marzbani et al., 2016). Therefore, this 
study was aimed at showing the impact of neurofeedback on a person's capability to control his or her brain functions.

In this study, the participants were asked to drive a car (like in a video game) through the brain waves captured by EEG. The car would run straightly and fast when the brain was in a certain brain wave. The participants were trained to control their brain waves in this process.

From a series of those neurofeedback processes, all of the participants were expected to be able to know the right brain wave that they should produce in a certain situation, for instance, alpha waves that could make a person relax or beta waves that could make a person focused and alert. Moreover, the participants were expected to be able to control their brain activities, thus the brain waves that were produced could enhance their leadership effectiveness.

\section{Conclusion}

The study conducted for four months and the results showed that all of the participants involved had the transformational leadership type with idealized influence (behavior) and inspirational motivation subtypes. Based on various literature studies, transformational leadership type is relatively more effective than transactional leadership type. Transformational leadership can enhance the employees'/followers' work satisfaction. It is more possible for an organization to achieve its goals with a high level of work satisfaction.

Moreover, transformational leadership is needed very much since it is a type of leadership that is not only able to motivate and move an organization vertically and horizontally but also is able to realize the organizational capacity to always be agile towards changes and dynamics according to the need. Therefore, in order to anticipate challenges in this VUCA era, an organization is in need of a flexible and adaptive leader since the world is changing rapidly. A leader should always learn and renew the organizational visions and strategies to keep up with the progress of the times. A transformational leader must possess flexibility and adaptability. Therefore, as a future leadership style, it can be noted that transformational leadership will eventually be able to transform the organizational paradigms and values to achieve its vision and missions optimally.

Another conclusion that we could draw from this study was that there were no different levels of intelligence among the participants (above average). Therefore, the brain activity patterns recorded with electroencephalography (EEG) showed the same patterns too. We then concluded that there was a relation between the level of intelligence and the leadership type.

Moreover, the presence of stimulation on the brain (neurofeedback) also made the participants able to control their brain waves consciously. Delta waves could be captured by EEG when a person sleeps, theta waves appear when a person is sleepy, alpha waves appear when a person feels relaxed and his or her muscles are not tense when he or she is conscious, beta waves appear when a person is conscious, while gamma waves appear when a person is solving a problem. When a leader can control his or her brain waves, he or she is expected to perform optimally.

The same patterns in the leadership type, level of intelligence, the brain activity mapping obtained from the study very probably resulted from the same environment of all participants. Therefore, to enrich our understanding on neuroscience-based and behavior-based leadership, we need to vary the samples that will be researched. One thing that we can do is that we 
involve participants from different departments or organizations. Moreover, regardless of a claim stating that analytically, this study greatly contributes to the application of sciences in the fields of leadership, especially the mapping on applied neuroscience transmission work mechanism in affecting leadership behaviors. It is important to further validate that claim especially when certain stimuli are given such as a stressful workplace in real-time situation.

\section{Reference}

Augustine, G. J. (2004). Studying of nervous systems of humans and other animals. In P. D. Augustine, G. J. Fitzpatrick, D. Hall, W. C. Lamantia, A. S. Mcnamara, \& J. O. Williams (Eds.), Neuroscience. Massachutes: Sinauer Associates.

Avolio, B. J., \& Bass, B. M. (1991). The multifactor leadership questionnaire: Form 5x. center for leadership studies. Binhampton, NY: State University of New York.

Axon, L., Friedman, E., \& Jordan, K. (2015). Leading now: Critical capabilities for a complex world. Boston: Harvard Business Publishing.

Bass, B. M. (1990). From transactional to transformational leadership: learning to share the vision. Organizational Dynamics, $18,19-31$

Bass, B. M., \& Avolio, B. J. (1994). Improving organizational effectiveness through transformational leadership. California: Sage Publications.

Bass, B. M., \& Bass, R. (2008). The Bass handbook of leadership: Theory, research, and managerial applications. New York: Simon and Schuster.

Benjamin, L., \& Flynn, F. J. (2006). Leadership style and regulatory mode: Value from It? Organizational Behavior and Human Decision Processes, 100, 216-230.

Berson, Y., \& Linton, J. D. (2005). An examination of the relationships between leadership style, quality, and employee satisfaction in $\mathrm{R} \& \mathrm{D}$ versus administrative environments. R\&D Management, 35, 51-60.

Boyatzis, R. E., Smith, M. L., \& Blaize, N. (2006). Developing sustainable leaders through coaching and compassion. Academy of Management Learning and Education, 5, 8-24.

Bradley, J. H., \& Hebert, F. J. (1997). The effect of personality type on team performance. Journal of Management Development, 16, 337-353.

Burns, J. M. (1978). Leadership. New York: Harper \& Row.

Cacioppo, J. T., Berntson, G. G., Lorig, T. S., Norris, C. J., Rickett, E., \& Nusbaum, H. (2003). Just because you're imaging the brain doesn't mean you can stop using your head: A primer and set of principles. Journal of Personality and Social Psychology, 85, 650-661.

Cacioppo, J. T., Berntson, G. C., \& Nusbaum, H. C. (2008). Neuroimaging as a new tool in the toolbox of psychological science. Current Directions in Psychological Science, 17, 62-67.

Case, R. (1992). The mind's staircase: exploring the conceptual underpinnings of children's thoughts and knowledge. Hillsdale, NJ, US: Lawrence Erlbaum Associates, Inc.

Charan, R. (2009). Leadership in the era of economic uncertainty: Managing in a downturn. New York: McGraw-Hill.

Cherif, A., Roelands, B., Meeusen, R., \& Chamari, K. (2016). Effects of intermittent fasting, caloric restriction, and Ramadan intermittent fasting on cognitive performance at rest and during exercise in adults. Sports Medicine, 46, 35-47.

Craig, A. D. (2009). How do you feel-now? The anterior insula and human awareness. National Review of Neuroscience, 10 , $59-70$

Damasio, A. R. (1989). Time-locked multiregional retroactivation: A systems-level proposal for the neural substrates of recall and recognition. Cognition, 33, 25-62.

Defoe, D. (2012). Leadership and brain science: possibilities for harnessing social cognitive neuroscience to assess, understand, and develop inspirational leaders. Psycholawlogy. Retrieved from https://www.psycholawlogy.com/2012/12/28/leadership-and-brain-science-possibilities-for-harnessing-social-cognitiveneuroscience-to-assess-understand-and-develop-inspirational-leaders/

Dempster, T. (2012). An investigation into the optimum training paradigm for alpha electroencephalographic biofeedback (Unpublished doctoral dissertation). Canterbury Christ Church University, Canterbury.

Evans J. R., \& Abarbanel, A. (1999). Introduction to quantitative EEG and neurofeedback. San Diego, California: Academic Press. 
Egner T., \& Gruzelier J. H. (2004). EEG Biofeedback of low beta band components: Frequency-specific effects on variables of attention and event-related brain potentials. Clinical Neurophysiology, 115(1), 131-139.

Fiedler, F. E., \& Garcia, J. E. (1987). New approaches to effective leadership: cognitive and organizational performance. United States: John Wiley \& Sons Inc.

Fuster, J. M. (1999). Memory in the cerebral cortex: an empirical approach to neural networks in the human and nonhuman primate. Cambridge: MIT Press.

Garret, B. (2003). Brain and behavior. Singapore: Thomson Learning.

Gomez-Pinilla, F., \& Hillman, C. (2013). The Influence of Exercise on Cognitive Abilities. Comprehensive Physiology, 3, 403-428.

Gruzelier, J. (2009). A theory of alpha/theta neurofeedback, creative performance enhancement, long distance functional connectivity and psychological integration. Cognitive Processing, 10(1), 101-109.

Haas, L. (2001). Phineas gage and the science of brain localisation. Journal of Neurology, Neurosurgery, and Psychiatry, 71, 761.

Hannah, S. T., Woolfolk, L., \& Lord, R. G. (2009). Leader self-structure: A framework for positive leadership. Journal of Organizational Behavior, 30, 269-290.

Harter, J. K., Smith, F. L., \& Hayes, T. L. (2002). Business-unit-level relationship between employee satisfaction, employee engagement, and business outcomes: A meta-Analysis. Journal of Applied Psychology, 87, 268-279.

Heinrich, H., Gevensleben, H. \& Strehl, U. (2007). Annotation: Neurofeedback - train your brain to train behavior. Journal of Child Psychology and Psychiatry, 48, 3-16.

Hollander, E. P. (1984). Leadership dynamics: A practical guide to effective relationships. New York: Free Press.

Homan, R., Herman, J., \& Purdy, P. (1987). Cerebral location of international 10-20 system electrode placement. Electroencephalography and Clinical Neurophysiology, 66, 376-382.

House, R. J., \& Aditya, R. N. (1997). The social scientific study of leadership: Quo Vadis? Journal of Management, 23, 409473.

Hunt, A., \& Wheeler, B. (2017). Brexit: All you need to know about the UK leaving the EU. BBC News, 25.

Juhro, S. M., \& Aulia, A. F. (2017). 2017. Transformational leadership through applied neuroscience: Transmission mechanism of the thinking process. BI Institute Working Paper, Bank Indonesia Institute - Bank Indonesia, Semester I/2017. Retrieved from https://ssrn.com/abstract=3015686

Jung, D. I. (2001). Transformational and transactional leadership and their effects on creativity in groups. Creativity Research Journal, 13, 185-195.

Jurkiewicz, C. E. (2000). Generation X and the public employee. Public Personnel Management, 29, 55-74.

Kolb, B., \& Whishaw, I. Q. (2011). An introduction to brain and behavior. United Kingdom: Palgrave.

Kirkpatrick, S. A., \& Locke, E. A. (1991). Leadership: Do traits matter? Academy of Management Executive, 5, 48-60.

Lawrence, J. A., Steck, E. N., \& Pryplesh, S. J. (1991). Overview of management theory. Pennsylvania: U.S. Army War College.

Lawrence, K. (2013). Developing leaders in a VUCA environment. Growbold, Retrieved from http://www.growbold.com/2013/developing-leaders-in-a-vuca-environment_UNC.2013.pdf.

Lewis, D. A. (1997). Development of the prefrontal cortex during adolescence: insights into vulnerable neural circuits in schizophrenia. Neuropsychopharmacology, 16(6), 385-398.

Liu, Y., Jing, Y., \& Gao, M. (2015). Transformational leadership: From the perspective of neurological leadership. Open Journal of Leadership, 4, 143-152.

Lieberman, M. D. (2007). Social cognitive neuroscience: A review of core processes. Annual Review of Psychology, 58, 259289.

Mann, R. D. (1959). A review of the relationships between personality and performance in small groups. Psychological Bulletin, 56, 241-270.

Marzbani, H., Marateb, H. R., \& Mansourian, M. (2016). Neurofeedback: A comprehensive review on system design, methodology, and clinical applications. Basic and Clinical Neuroscience, 7, 143-158.

Ming, G. L., \& Song, H. (2012). Adult neurogenesis in the mammalian brain: Significant answers and significant questions. Neuron, 70, 687-702.

Muessig, R. H. (1969). Change - the only constant. Educational Leadership, 26, 543-546. 
Murphy, T. H., \& Corbett, D. (2009). Plasticity during stroke recovery: From Synapse to behaviour. Nature Reviews Neurosciences, 10, 861-872.

Naylor, J. (2003). Management. United Kingdom: Financial Times / Prentice Hall.

Nolte, J. (2002). The human brain: An introduction to its functional anatomy. Missouri: Mosby.

Ochsner, K. N., \& Lieberman, M. D. (2001). The emergence of social cognitive neuroscience. American Psychologist, 56, 717-734.

Podsakoff, P. M., Bommer, W. H., Podsakoff, N. P., \& Mackenzie, S. B. (2006). Relationships between leader reward and punishment behavior and subordinate attitudes, perceptions, and behaviors: A meta-analytic review of existing and new research. Organizational Behavior and Human Decision Processes, 99, 113-142.

Rabin, A. I., \& Guertin, W. H. (1951). Research with Wechsler-Bellevue test. Psychological Bulletin, 48, 211-248.

Radha, R., \& Kosuri, S. R. (2017). The straits of success in a VUCA world. IOSR Journal of Business and Management, 1622.

Ros, T., Moseley, M. J., Bloom, P. A., Benjamin, L., Parkinson, L. A., Gruzelier, J. H. (2009). Optimizing microsurgical skills with EEG neurofeedback. BMC Neuroscience, 10(1), 87.

Rosenzweig, M. R., \& Bennet, E. L. (1999). Psychobiology of plasticity: Effects of training and experience on Brain and behavior. Behavioural Brain Research, 78, 57-65.

Rowold, J. (2005). Multifactor leadership questionnaire. Psychometric properties of the German translation by Jens Rowold. Redwood City: Mind Garden.

Salah, A., \& Watson, R. (2017). Business excellence in a volatile, uncertain, complex, and ambiguous environment (BEVUCA). The TQM Journal, 29, 705-724.

Salahuddin M. M. (2010). Generational differences impact on leadership style and organizational success. Journal of Diversity Management, 5(2), 1-5.

Sarin, S., \& Mcdermott, C. (2003). The effect of team leader characteristic on learning, knowledge application, and performance of cross-functional new product development teams. Decision Sciences, 34, 707-739.

Sarkar, A. (2016). We live in a VUCA world: The importance of responsible leadership. Development and Learning in Organizations: An International Journal, 30, 9-12.

Schore, A. N. (1994). Affect regulation and the origin of the self: the neurobiology of emotional development. Hillsdale, NJ, US: Lawrence Erlbaum Associates, Inc.

Siddiqui, S. V., Chatterjee, U., Kumar, D., Siddique, A., \& Goyal, N. (2008). Neuropsychology of prefrontal cortex. Indian Journal of Psychiatry, 50, 202-208.

Sierra, A., Encinas, J. M., \& Maletic-Savatic, M. (2011). Adult human neurogenesis: From microscopy to magnetic resonance imaging. Frontiers in Neuroscience, 5, 1-18.

Stiehm, J. H. (2002). U.S. army war college: Military education in a democracy. Philadelphia, Pennsylvania, United States: Temple University Press.

Stogdill, R. M. (1948). Personal factors associated with leadership: A survey of the literature. The Journal of Psychology, 25, $35-71$.

Stone, J., \& Hughes, J. (2013). Early history of electroencephalography and establishment of the American clinal neurophysiology society. Journal of Clinical Neurophysiology, 30, 28-44.

Staufenbiel, S., Brouwer, A. M., Keizer, A., \& Van Wouwe, N. (2014). Effect of beta and gamma neurofeedback on memory and intelligence in the elderly. Biological Psychology, 95, 74-85.

Surmeli T., \& Ertem A. (2007). EEG neurofeedback treatment of patients with Down Syndrome. Journal of Neurotherapy, 11(1), 63-68.

Sucholeiki, R. (2017). Normal EEG waveforms [Online]. Medscape, Retrieved from https://emedicine.medscape.com/article/1139332-overview.

Tolbize, A. (2008). Generational differences in the workplace. Research and Training Center on Community Living, 5(2), 121.

Tovar, P. (2016). Leadership challenges in the V.U.C.A world [Online]. Oxford Leadership, Retrieved from http://www.oxfordleadership.com/leadership-challenges-v-u-c-world/.

Tudor, M., Tudir, L., \& Tudor, K. (2005). Hans Berger (1873-1941) - the history of electroencephalography. Acta Medica Croatia, 59, 307-313. 
Vernon D. J. (2005). Can neurofeedback training enhance performance? An evaluation of the evidence with implications for future research. Applied Psychophysiology Biofeedback, 30(4), 347-364.

Vince, R. (2004). Rethinking strategic learning. United Kingdom: Routledge.

Waldman, D. A., Balthazard, P. A., \& Peterson, S. J. (2011). Leadership and neuroscience: Can we revolutionize the way that inspirational leaders are identified and developed? Academy of Management Perspectives, 25, 60-74.

Weiherl, J., \& Masal, D. (2016). Transformational leadership and follower's commitment to mission changes. International Journal of Public Administration, 39, 861-871.

$\mathrm{Xu}, \mathrm{G}$. Y., \& Wang, Z. S (2008). The impact of transformational leadership style on organizational performance: The intermediary effect of leader-member exchange. Paper presented at the International Conference on Management Science and Engineering, Long Beach, CA, USA, IEEE Xplore.

Yammarino, F. (2013). Leadership: Past, present, and future. Journal of Leadership and Organizational Studies, 20, $149-155$.

Zemke, R., Raines, C., \& Filipczak, B. (2000). Generations at work: Managing the clash of veterans, boomers, Xers, and Nexters in your workplace. New York: Amacom. 